Miller Rivera Lozano*

Nicolás Rivera Garzón

Recibido: 12 de noviembre de 2018

Concepto de evaluación: 5 de diciembre de 2018

Aprobado: 8 de febrero de 2019

Artículo de investigación

(c) 2019 Universidad Católica de Colombia.

Facultad de Ciencias

Económicas y Administrativas.

Todos los derechos reservados

\title{
Crisis financieras: esta vez no es distinto
}

\section{RESUMEN}

Este artículo presenta una discusión sobre las principales características teóricas de las diferentes tipologías de crisis financieras. Para ello, se hace una explicación detallada del funcionamiento del sistema financiero moderno, la historia reciente de la macroeconomía y las propiedades conceptuales y empíricas de las crisis financieras. Se concluye que un banco central está limitado para actuar contra burbujas de crédito y que los modelos macroeconómicos actuales no pueden explicar correctamente las interacciones dentro los mercados financieros. Asimismo, se deduce la posibilidad de que las economías emergentes en el ámbito mundial sufran crisis financieras en los tiempos próximos.

Palabras clave: burbuja especulativa, crisis financieras, economías emergentes, macroeconomía, sistema financiero.

JEL: E44, E58, G10, G28.

Cómo citar este artículo / To reference this article / Para citar este artigo:

Rivera Lozano, M., \& Rivera Garzón, N. (2019). Crisis financieras: esta vez no es distinto. Revista Finanzas y Política Económica, 11 (1), 129-147. doi:http://dx.doi. org/10.14718/revfinanzpolitecon.2019.11.1.8

\section{Financial crises: It is not different this time}

\section{ABSTRACT}

This article discusses the main theoretical characteristics of several

*Doctor en educación (c). Docente e investigador de la Universidad Santo Tomás,

Facultad de Administración de Empresas. Bogotá, Colombia

Correo: millerrivera@usantotomas.edu.co

(D) http://orcid.org/0000-0001-5257-8400

** Economista (c). Facultad de Ciencias Económicas, Universidad Nacional de Colombia, Bogotá. Correo: nriverag@unal.edu.co. (D) http://orcid.org/0000-0002-0044-5435 types of financial crises. With this objective in mind, it presents a detailed explanation of the functioning of the modern financial system, recent history of macroeconomics, and the conceptual and empirical properties of financial crises. It concludes that a central bank is limited to act against credit bubbles and that current macroeconomic models cannot explain correctly interactions in financial markets. In addition, it deduces the possibility that emerging economies worldwide will experience financial crisis in the coming months.

Keywords: Financial crises, financial system, macroeconomics, speculative bubble, emerging economies. 


\section{Crises financeiras: desta vez não é diferente}

\section{RESUMO}

Este artigo apresenta uma discussão sobre as principais características teóricas dos diferentes tipos de crises financeiras. Com esse objetivo em mente, uma explicação detalhada é dada sobre o funcionamento do sistema financeiro moderno, a história recente da macroeconomia e as propriedades conceituais e empíricas das crises financeiras. Dessa forma, conclui-se que um banco central é limitado a agir contra as bolhas de crédito e que os modelos macroeconômicos atuais não conseguem explicar corretamente as interações dentro dos mercados financeiros. Além disso, deduz-se que economias emergentes em todo o mundo sofram crise financeira nos próximos meses.

Palavras-chave: crises financeiras, sistema financeiro, macroeconomia, bolha especulativa, economias emergentes. 


\section{INTRODUCCIÓN}

La esencia del síndrome de "esta vez es distinto" es simple. Se basa en la firme creencia de que una crisis financiera es algo que les sucede a otras personas en otros países en otras épocas: las crisis no nos afectan a nosotros, aquí y ahora. Estamos haciendo bien las cosas, somos más inteligentes, hemos aprendido de los errores del pasado.

Reinhart y Rogoff (2011, p. 30)

El síndrome de "esta vez es distinto" hizo su primera aparición en el siglo XXI con la crisis de las hipotecas subprime en el 2008, que desembocó en el episodio conocido como la Gran Recesión. La economía estadounidense sostuvo una extraordinaria secuencia de grandes déficits en cuenta corriente ${ }^{1}$ antes de explotar la crisis del 2008. El déficit norteamericano explicaba más de $2 / 3$ del superávit en cuenta corriente del mundo (Reinhart y Rogoff, 2008), y por si esto fuera poco, el precio de la vivienda se duplicó. La economía estadounidense mostraba todas las señales de una crisis financiera próxima, pero ni los mejores economistas de mundo pudieron evitarla (Chorafas, 2013).

La crisis anterior está lejos de ser la única en las economías avanzadas; las más destacadas conforman el grupo de "las cinco grandes crisis"; España (1977), Noruega (1987), Finlandia (1991), Suecia (1991) y Japón (1992) forman parte de este grupo. Todas ellas fueron prolongadas y de gran escala, y estuvieron asociadas a un gran deterioro del desempeño económico durante un periodo extenso (Reinhart y Rogoff, 2008). Y pese a que estas se hayan dado, el sistema financiero se ha profundizado cada vez en las economías modernas y su desarrollo es cada vez más importante.

1 La cuenta corriente de la balanza de pagos es una sección que resume todas las transacciones por concepto de exportaciones e importaciones de bienes y servicios. Esta se divide en cuenta de bienes y servicios, ingreso primario y secundario. El saldo de esta cuenta equivale a la diferencia entre exportaciones e ingreso por recibir y las importaciones e ingreso por pagar.
El desarrollo financiero es un proceso para reducir los costos de adquisición de información, cumplimiento de contratos y realización de transacciones (Banco Mundial, 2018). Sin embargo, en las crisis más recientes de economías avanzadas tal desarrollo ha tomado la forma de una marcada liberalización de los mercados financieros y de sus marcos regulatorios. La liberalización financiera dio origen a sectores financieros nacionales caracterizados por quiebras generalizadas, intervenciones gubernamentales masivas y un bajo nivel de ahorro interno (Reinhart, 2015; Lorente, 2018). Los economistas pueden o no estar de acuerdo con la noción según la cual la liberalización financiera es la antesala de cualquier crisis en el sistema financiero, pero coinciden en que son muy costosas para la sociedad. Reinhart y Rogoff (2009) muestran por ejemplo que en los principales episodios posteriores a la Segunda Guerra Mundial el valor real de la deuda del gobierno aumentó en promedio $86 \%$. De este modo, las naciones o regiones que atraviesan por una crisis financiera viven las consecuencias por muchos años más de los que comúnmente se cree, como lo evidencia Blanchard (1997) en la teoría de la histéresis pura del desempleo.

Este artículo se compone de cuatro secciones, además de esta introducción. La primera hace una breve exposición sobre el funcionamiento general de un sistema financiero y de las relaciones que tienen los agentes dentro de él. La segunda discute el papel de la macroeconomía y los modelos macroeconómicos en los análisis del sistema financiero. En la tercera se abordan las principales características teóricas de las diferentes tipologías de crisis financieras, destacando dentro de estas la liberalización como una de sus causas.

Jonung y Hagberg (2005) exponen que el costo de una crisis para la sociedad en su conjunto se debe medir en términos de pérdida de producto o pérdida de crecimiento del producto. Los costos que trae consigo una crisis se expondrán en la última sección. 


\section{CONCEPTOS FUNDAMENTALES}

\section{Sistema financiero}

Antes de introducir los fundamentos que dan origen a una crisis bancaria o crisis de deuda interna o externa, es necesario entender qué es un sistema financiero y qué agentes interactúan en su interior. Un sistema financiero es esencialmente la unión de las instituciones financieras y no financieras de un país, los mercados financieros (monetario, cambiario y de crédito), sus interacciones y su infraestructura financiera (Banco Mundial, 2018). Las empresas financieras o bancos basan su operación en llevar al máximo su margen de intermediación, es decir, buscan captar ahorro financiero a la menor tasa de interés posible y colocarlo en el mercado a una tasa de interés más alta.

De su actividad se puede deducir simplificadamente cómo se divide el balance general de cualquier banco comercial ${ }^{2}$. En sus pasivos se encuentran las operaciones de captación de recursos o custodia de dinero de familias, empresas no financieras y el gobierno (estos son los depósitos bancarios). En sus activos se encuentran los créditos a los demás agentes de la economía y la reserva bancaria en posesión del banco central. Los bancos tienen un papel clave en cualquier economía: son los encargados de mantener un flujo constante de liquidez para hacer inversiones, gasto privado o gasto público. Desde su mera definición, ya es evidente que su mal funcionamiento puede poner en aprietos a cualquier país muy rápidamente, tema que será abordado con mayor profundidad en las siguientes secciones.

Dentro de los agentes no financieros se van a agrupar las empresas no financieras, los hogares y el gobierno. Las empresas no financieras realizan dos funciones dentro del sistema financiero: tienen

2 Cuando un banco otorga un crédito, está creando nuevos medios de pago. Esta operación la hace al registrar en su contabilidad un pagaré en la sección de activos y, en simultáneo, un valor en la cuenta corriente del cliente, es decir, en sus pasivos. depósitos y buscan financiamiento; para decidir entre ahorrar o invertir, calcularán un costo de oportunidad traducido en la tasa de interés de mercado vigente. Si la tasa de interés de mercado es baja, tendrán incentivos para pedir financiamiento; si es alta, pondrán su exceso de liquidez o ahorro financiero en los bancos en forma de depósito. Los hogares operan de la misma forma que las empresas no financieras; la mayor diferencia es que las empresas piden financiamiento para invertir y no para consumir, mientras los hogares hacen ambos.

El último agente no financiero que desempeña un rol importante es del gobierno. Este interactúa con los demás agentes del sistema financiero como regulador y como usuario del sistema (Hernández, 2005): como regulador, determina y vigila las reglas que los demás agentes deben cumplir; como usuario tiene depósitos y pide financiamiento, o en otras palabras, su función es hacer política fiscal. Aquí surgen conflictos entre la política fiscal del gobierno y la del banco central -que es un organismo autónomo-. Un ejemplo claro de este conflicto es la dominancia de la política fiscal a la política monetaria en tiempos de recesión. Cuando un gobierno está muy cerca de entrar en situación de default, la consecuencia más próxima será el colapso del valor de la moneda doméstica. Este colapso lleva a una inflación (Svensson, 2000; Blanchard, 2018) más alta y a una peor contracción económica; por tanto, el banco central se verá obligado a monetizar la deuda para evitar tal situación (Mishkin, 2017).

Los agentes se enfrentan a la disyuntiva de elegir mantener dinero en efectivo o mantener bonos; por eso, producto de esta situación, es necesario tener claridad conceptual sobre ambos activos para después explicar detalladamente esta elección. El dinero es el activo más líquido que existe y puede moverse y repartirse en cualquier espacio económico. Las cuatro funciones principales del dinero son: unidad de cuenta, medio de pago, reserva de valor y patrón para pagos diferidos (vincula por medio de contratos financieros valores actuales y futuros). Estas cualidades hacen que el dinero sea un activo para el poseedor y un pasivo para el banco 
central que lo emitió, debido a que la propiedad de liquidez le da al poseedor rendimientos (flujos), poder de compra generalizado y capacidad para honrar sus obligaciones financieras de inmediato (poder liberatorio). Además, existen dos tipos de dinero: efectivo (monedas y billetes) y depósitos a la vista (depósitos bancarios) (Blanchard, Amighini y Giavazzi, 2012).

Por otro lado, un bono es un instrumento financiero que ofrece una promesa de pagar al propietario una rentabilidad determinada; puede ser emitido por el Estado, empresas no financieras y empresas financieras en busca de financiamiento en los mercados financieros. Según Blanchard et al. (2012), los bonos se diferencian en dos aspectos básicos: el riesgo de impago y el plazo. La principal motivación para mantener riqueza en forma de bonos es que estos rinden un interés determinado; en otra situación los agentes preferirían mantener efectivo.

Teniendo en cuenta estas dos definiciones anteriores, ahora es posible ahondar en la discusión sobre el funcionamiento de la demanda de bonos y de dinero. Por un lado, la teoría cuantitativa de la demanda de dinero ${ }^{3}$ argumenta que existe una relación marginal de sustitución decreciente entre el dinero y los bonos; es decir, a medida que el rendimiento de los bonos aumenta, los agentes cada vez más optan por tener su riqueza de esta forma. Desde el punto de vista keynesiano (Blanchard et al., 2012) sucede algo similar: los agentes tienen una demanda de dinero, motivo especulativo en la cual se "apuesta" cuál va a ser la tasa de interés real de mercado en determinado periodo. Entonces la relación inversa entre el precio del bono y las tasas de interés afecta la demanda de dinero. La explicación anterior, aunque es una simplificación de la forma en que los individuos toman decisiones en el mercado financiero, sirve de guía para entender la importancia que tienen los bonos y el dinero dentro de este conjunto de mercados.

$3 \quad$ La demanda real de dinero depende del nivel de riqueza (el dinero es un bien normal), el capital humano, el nivel de precio, la aceleración de la inflación, el rendimiento de los demás activos en la economía y el flujo de salarios esperados.
Claramente, los sistemas financieros domésticos están insertos en el sistema financiero global. Los bancos globales son los principales agentes dentro de este sistema. Un banco global o internacional es un banco con activos de gran tamaño y con un alcance comercial internacional (Banco Mundial, 2018). Los bancos domésticos y los bancos internacionales se comportan de manera similar: ambos reciben préstamos, inversiones de capital, inyecciones de capital o préstamos interbancarios. De igual forma, ambos están obligados al pago de dividendos y a la transferencia de beneficios a los inversionistas o a su casa matriz.

Por todas las relaciones anteriores, la probabilidad de contagio de una crisis financiera entre países es cada vez mayor. La globalización y el fortalecimiento de la informática han permitido que el mundo se convierta en una aldea global donde se comparte conocimiento y beneficios a una velocidad nunca vista; sin embargo, esta velocidad también ha propiciado que las consecuencias de cualquier revés en el comercio o en el sistema financiero de una nación se expandan por muchas regiones. Lo anterior explica por qué las soluciones aisladas para salir de una crisis financiera global no son efectivas, pues ante cualquier crisis financiera venidera, el mundo debe entender que se necesitan medidas en el ámbito mundial ${ }^{4}$, no solo en el nacional:

Es evidente que la crisis actual ha alterado de manera profunda la índole de la competencia mundial y que tenemos que reconsiderar todos los elementos de las reglas del juego para poder salir adelante. Se trata de una crisis verdaderamente mundial y, como la economía está globalizada, el problema sólo se puede resolver en el plano mundial. (Stiglitz, 2009, p. 4)

4 Después de la crisis de 2008, la política monetaria mundial tuvo un periodo de diez años seguidos con tasas bajas de interés y una expansión monetaria constante para reactivar la economía mundial. 


\section{Macroeconomía}

Prescott (2006) dice que la macroeconomía se ha vuelto como las ciencias naturales; su comentario está enfocado en la metodología que usa la macroeconomía, y no en el objeto de estudio de cada ciencia. La macroeconomía moderna se ha apoyado explícitamente en el razonamiento lógico, la matemática, la estadística y la probabilidad; en otras pablaras, intenta acercarse cada vez más al uso completo del método científico. Aunque metodológicamente el estudio de la economía agregada se parece cada vez más a una ciencia "dura", el objeto de estudio de la economía siempre serán las relaciones complejas entre individuos y sus interacciones en el mercado y la producción. Debido a ello, nunca habrá un consenso total en la profesión sobre la teoría que se debería usar; solo hay consenso sobre el objeto de estudio, como argumentan Snowdon y Vane (2005):

La macroeconomía se refiere a la estructura, el rendimiento y el comportamiento de la economía en su conjunto. La principal preocupación de los macroeconomistas es analizar e intentar comprender los determinantes subyacentes de las principales tendencias agregadas de la economía con respecto a la producción total de bienes y servicios (PIB), el desempleo, la inflación y las transacciones internacionales. En particular, el análisis macroeconómico busca explicar la causa y el impacto de las fluctuaciones a corto plazo en el PIB (el ciclo económico) y los principales determinantes del camino a largo plazo del PIB (crecimiento económico). (p. 1)

Con la preocupación anterior en mente, los economistas han desarrollado modelos para tener un marco común de análisis y de intervención de la realidad; sin embargo, ha sido necesario introducir simplificaciones en estos constructos teóricos debido a la complejidad de las relaciones sociales existentes en la sociedad. Estas han tomado la forma de supuestos que condicionan el comportamiento y los resultados del modelo a cambio de mayor claridad y sencillez; la dificultad de este asunto surge cuando los diseñadores de políticas asumen algo como cierto y lo defienden a toda costa para escudarse de las fallas en la teoría. Las verdades evidentes en las políticas públicas han estado en el centro de los recientes debates académicos y políticos (Ostrom, 2000; Raworth, 2017). Los economistas deben superar estas verdades evidentes para responder a las preguntas más importantes de la sociedad, y esto implica realizar un análisis detallado de los aspectos centrales de los modelos de equilibrio general usados en la política pública.

Los supuestos y metodologías usados tienen ventajas y desventajas; por ejemplo, cuando se usan agentes representativos para hacer análisis económicos, se sacrifica la posibilidad de analizar las interacciones complejas entre agentes por un análisis más rico de la maximización intertemporal (Stiglitz y Gallegati, 2011). De lo anterior se puede concluir erróneamente que los modelos usados en política económica tienen variedad de enfoques y teorías, pero lastimosamente ese no es el caso. Los modelos de equilibrio general se han vuelto cada vez más homogéneos, característica que merma su capacidad analítica. Además, los modelos usados en política económica no se han producido a una escala, nivel de detalle y ajuste que les permita ser utilizados en el proceso real de formación de política monetaria y fiscal (Sims, 1996; Lorente, 2018).

Durante los sesenta, el modelo macroeconométrico keynesiano básico fue la teoría establecida, que no solo incorporaba la teoría keynesiana, sino que la articulaba con los postulados neoclásicos y estaba fuertemente inspirada en el modelo de crecimiento de Robert Solow. A este respecto, Snowdon y Vane (2005) argumentan que la síntesis neoclásica keynesiana estaba sustentada en la combinación de tres elementos: a) modelos macroeconómicos que lleven a cabo optimización intertemporal, b) uso de la hipótesis de expectativas racionales y reconocimiento de competencia imperfecta ${ }^{5}$ en los mercados de bienes, trabajo y crédito, c) incorporación de precios de ajuste en los 
modelos macroeconómicos. Además, el modelo era capaz de realizar una compensación entre inflación y desempleo (Prescott, 1995, 2002; Solow, 2004). Sin embargo, la fuerza de este modelo llegó a su fin con la crítica de los años setenta (para entender la contrarrevolución neoclásica, véase Snowdon y Vane, 2005). Lucas (1976) demostró dos problemas con el pronóstico macroeconómico y la evaluación de políticas: a) no existen fundamentos teóricos para las macroecuaciones del modelo; b) el modelo macroeconométrico keynesiano fracasó en los setenta justamente como la teoría monetarista predijo (estanflación de los años setenta).

Años más tarde, las investigaciones teóricas llegaron a la conclusión de que la crítica de Lucas (1976) afectaba a cualquier modelo macroeconométrico (Haldane y Turrell, 2017), pero el paradigma keynesiano ya había sido desplazado, la síntesis neoclásica keynesiana mutó. Blanchard (1997) argumenta que la macroeconomía moderna se basa en las dos siguientes proposiciones: a) en el corto plazo, los movimientos en la actividad económica están dominados por movimientos en la demanda agregada; b) con el tiempo, la economía tiende a volver a una senda de crecimiento en estado estacionario.

La combinación de las proposiciones anteriores ofrece una caracterización compleja de los efectos de los shocks y de la política macroeconómica; sin embargo, requiere que los agentes tengan expectativas racionales. Con la condición anterior, la economía reacciona de una manera muy singular ante cualquier perturbación. Lorente (2018) muestra dos tipos de reacciones: a) si sucede una perturbación imprevista, la racionalidad exige que los agentes salten inmediatamente a la nueva trayectoria convergente; b) si la perturbación es previsible en algún momento anterior a su ocurrencia, los agentes se moverán a una trayectoria inicialmente divergente, pero después de ello será convergente a un estado estacionario. La presencia de expectativas racionales en cualquier modelo implica que los agentes tienen capacidad de cálculo infinita, perfecta información en todo momento, total conocimiento del sistema económico y no cometen errores; la combinación de estas capacidades hace que el agente representativo del modelo siempre elija la combinación de capital y consumo que esté dentrol de la trayectoria óptima y estable de la economía.

El resultado del debate teórico y metodológico de estas décadas fue el modelo de equilibrio general dinámico estocástico (EGDE), definido por Solow (2008) de este modo:

Modelo en el que un único consumidortrabajador-propietario inmortal maximiza una función de utilidad de tiempo aditivo perfectamente convencional en un horizonte infinito, bajo previsión perfecta o expectativas racionales, $y$ en un contexto institucional, y el entorno tecnológico que favorece el comportamiento universal de toma de precios. (p. 243).

Estos modelos tienen un equilibrio único con pequeñas desviaciones, sin papel para las variables bursátiles, y los microcomportamientos de los agentes pueden agregarse de manera simple y lineal en un agente representativo con expectativas racionales y con racionalidad perfecta (Haldane y Turrell, 2017).

A partir de lo anterior se puede llegar a una conclusión alarmante: el modelo EDGE con agentes representativos y expectativas racionales no tiene nada qué decir acerca del funcionamiento del sistema financiero; por ende, no tiene la capacidad de dar consejos a los diseñadores de política sobre cómo superar una crisis financiera. Stiglitz y Gallegati (2011) nombran tres limitaciones que tiene el enfoque del agente representativo a la hora de analizar los shocks en la red de relaciones de crédito: a) el choque que da lugar a la fluctuación macroeconómica es uniforme entre los agentes; b) la visión agregada del agente representativo no puede capturar el hecho de que la propagación de una enfermedad financiera puede avanzar a diferentes velocidades en diferentes partes de la macroeconomía; c) este enfoque no logra asimilar que, en una red de crédito, una quiebra de banco puede provocar una avalancha de bancarrota. 
La mayor justificación para usar el agente representativo con racionalidad perfecta y expectativas racionales era que satisfacía un conjunto de microfundamentos estrictos (Kirman, 1989; Thaler, 2000; Colander et al., 2008; Raworth, 2017; Blanchard, 2018); no obstante, el teorema de Sonnenschein-Mantel-Debreu (SMD) debilitó tal premisa, pues mostró que las hipótesis que garantizan un buen comportamiento a nivel microeconómico no transfieren un buen comportamiento a nivel macroeconómico (Monsalve, 2017).

El nuevo paradigma que se alza como una solución al enfoque del agente representativo son los modelos que combinan diferentes tipos de agentes con cierto grado de heterogeneidad. Los modelos basados en agentes (en inglés, agentbased models [ABM]) se fundamentan en el estudio computacional de agentes en constante evolución e interacción con su ambiente. Ahn, Ostrom y Walker (2003) y Janssen y Ostrom (2006) argumentan que los $A B M$ han tomado un lugar más central en las discusiones teóricas debido a tres ventajas sobre los modelos comunes: a) la creciente investigación teórica ha logrado desarrollar metodologías técnicas válidas para el proceso de modelación; b) actualmente hay más disponibilidad de datos para trabajar; c) la economía experimental ha mostrado las dificultades de los modelos convencionales y ha permitido un mayor espacio para los ABM en el estudio de dilemas sociales y conflictos en la toma de decisiones. A estas tres ventajas también se le puede sumar un tratamiento más especializado de los datos:

Distribuciones no gaussianas, ecuaciones no lineales, elecciones inconsistentes en el tiempo, comportamientos acotados racionales: es posible resolver todos estos numéricamente a nivel de agente. Dado que muchos problemas del mundo real involucran estas características, los ABM tienen muchos usos potenciales en economía. (Haldane y Turrell, 2017, p. 15)

\section{Un marco teórico sobre crisis financieras}

Bordo et al. (2001) definen una crisis financiera en los mercados financieros como episodios de alta volatilidad marcados por un alto grado de problemas de liquidez e insolvencia entre los participantes. Una crisis financiera se puede clasificar como una crisis bancaria, una crisis de deuda interna o externa y crisis cambiarias. En esta sección se desarrollará un marco teórico sobre las principales características de las tipologías de crisis financieras que existen y cuáles son los factores que las anteceden. Dentro de estos factores se destaca la liberalización financiera. Las crisis bancarias son menos probables en presencia de controles de capital (Bordo et al., 2001). En último lugar, se expondrá un marco teórico sobre burbujas de crédito y contagios financieros. La autoridad monetaria es la primera que se ve enfrentada a cualquier tipo de burbuja económica, pero, como se demostrará adelante, no tiene muchas herramientas para luchar contra esta. Las crisis tienden a desarrollarse en cámara lenta (Kaminsky, Reinhart y Végh, 2003); a las autoridades económicas solo les queda observar y limpiar las consecuencias después.

\section{Tipologías de las crisis financieras}

Jonung y Hagberg (2005) definen una crisis financiera como una fuerte caída de la actividad económica. Cuanto más caiga el ingreso (PIB), más profunda será la crisis. Debido a la complejidad del sistema financiero, los diferentes tipos de crisis financieras, como se señaló, pueden clasificarse en crisis bancarias, crisis de deuda interna o externa y crisis cambiarias. De lo anterior no se puede concluir que cada tipo de crisis sea excluyente de las demás; de hecho, usualmente una crisis financiera es provocada por la combinación de dos o más tipologías de crisis ya mencionadas.

Inicialmente, Reinhart y Rogoff (2011) muestran que, en promedio, la deuda pública se 
incrementa $86 \%$ en los tres años que siguen a una crisis bancaria. También identifican una crisis de este tipo por dos tipos de eventos:

1) Pánicos bancarios que conducen al cierre, fusión o adquisición por parte del sector público de una o más instituciones financieras, y 2) si no hay pánicos, el cierre, fusión, adquisición o apoyo a gran escala por parte del gobierno de una institución financiera importante (o grupo de instituciones), dando así inicio a una serie de desenlaces similares para otras instituciones financieras. (p. 41)

La estabilidad financiera no está asegurada con la estabilidad en el nivel de precio o de producto de la economía (Mishkin, 2017). Esta situación se da porque las interacciones complejas entre los agentes pueden generar tensiones en los mercados; es decir, un operador que trance divisas en el mercado cambiario puede estar tomando decisiones con base en sus expectativas y beneficio propios, aunque ello pueda afectar el comportamiento de otros agentes. Esta situación se asemeja conceptualmente a las cascadas de información: debido a la asimetría de la información en el sistema financiero, estas se producen cuando es óptimo para un individuo seguir el comportamiento del individuo anterior sin tener en cuenta la propia información (Kaminsky, Reinhart y Végh, 2003).

Esta situación es el detonante principal de una crisis cambiaria, también conocida como crisis de balanza de pagos. En este tipo de crisis se produce un ataque especulativo contra la moneda doméstica; los operadores del mercado cambiario comprarán grandes cantidades de moneda divisa, haciéndola escasa en el mercado. Este actuar se traducirá en una devaluación (tipo de cambio fijo) o depreciación (tipo de cambio flexible), según sea el caso. Los ataques especulativos son resultado de cambios en los sentimientos del mercado (Kaminsky y Reinhart, 1999). El banco central se verá así obligado a disminuir sus reservas internacionales mediante operaciones de mercado abierto ${ }^{5}$, endurecer su política monetaria para transmitir confianza a los mercados o, si es el caso, dejar que su moneda doméstica flote libremente. Las consecuencias de una crisis de esta índole son duraderas en cualquier economía. Para una crisis en balanza de pago, el crecimiento del producto en los doce meses siguientes está en un rango de $2 \%$ a $6 \%$ por debajo de las tasas de crecimiento comparables durante periodos tranquilos (Kaminsky y Reinhart, 1999).

Lo siguiente será tratar las crisis de deuda interna o externa. Reinhart y Rogoff (2011) definen un impago soberano como "el incumplimiento de un gobierno en el pago de crédito principal o de sus intereses antes de la fecha de vencimiento (o dentro del periodo acordado de gracia)" (p. 41). Una crisis o impago de deuda interna tiene las mismas características que una crisis o impago de deuda externa; sus principales diferencias son evidentemente la nacionalidad del acreedor y las deudas contraídas con agentes en el exterior, que están generalmente denominadas en moneda divisa (Blanchard, Amighini y Giavazzi, 2012; Hernández, 2005).

Cabe aquí aclarar que el episodio de impago externo no es solo de deuda pública, sino que también entra la deuda contraída por agentes privados, debido a que la balanza de pagos está conformada por todos los movimientos que hace una economía con el resto del mundo. De igual forma, aunque menos común, los episodios de impago interno también pueden ser provocados por incumplimientos en los pagos o intereses por parte de agentes privados a otros nacionales. Según lo anterior, la principal secuela de una crisis de deuda interna o externa es la reprogramación de la deuda bajo condiciones menos favorables que la obligación original (Reinhart y Rogoff, 2011).

Las crisis financieras suelen ser un mecanismo de amplificación de recesiones. Después de una crisis bancaria sistémica, en promedio pasan unos

5 Cuando un banco central hace cualquier tipo de operación de mercado abierto, debe "esterilizarla", es decir, compensar los efectos sobre la base monetaria de intervenir en el mercado de divisas. 
ocho años para alcanzar el nivel de ingreso anterior a la crisis (Reinhart y Rogoff, 2014). Una caída en el valor de los activos lleva a una disminución del consumo y de la inversión, que a su vez provocan una contracción económica. El sistema económico entra así en un círculo vicioso de recesión (Mishkin, 2017). Otro ejemplo puede ser el siguiente: por un bajo crecimiento del producto se incumplen créditos bancarios; dicha situación provoca que los otros bancos cancelen el otorgamiento de créditos; luego se dan caídas en la producción y nuevos episodios de impago, y así sucesivamente. Las consecuencias fiscales de una crisis financiera superan por mucho las estimaciones de los costos de rescate inicial.

\section{Gestación de una crisis}

Reinhart y Rogoff (2011) enuncian cuatro factores precursores clave de una crisis financiera: aumentos sostenidos de la deuda (pública o privada), grandes déficits en cuenta corriente, desaceleración de la actividad económica real y un aumento considerable en los precios de los activos. El deterioro en los fundamentos económicos de un país es la causa de las crisis externas o domésticas. Un deterioro en los fundamentos de una economía implica que las empresas - $y$ en muchos casos el gobierno- están en situación de insolvencia. Las empresas insolventes en economías de mercado se ven obligadas a vender activos importantes para obtener nuevos fondos (Caprio y Klingebiel, 1997). Este revés provoca que las entidades bancarias se vean obligadas a restringir nuevas oportunidades de crédito y a endurecer las condiciones de los créditos ya incumplidos. Además, si la empresa está cotizando en bolsa, los inversionistas no la verán como una oportunidad de inversión. Malas políticas, tanto microeconómicas (regulatorias) como macroeconómicas, y mala banca causan la mayoría de los episodios de insolvencia (Caprio y Klingebiel, 1997; Farhi y Tirole, 2018).

Cualquiera sea el caso, si gran cantidad de empresas enfrentan la misma situación, los bancos de dicha economía enfrentaran también problemas de insolvencia y no podrán proporcionar los créditos necesarios a otros agentes de la economía, y esta última entrará en recesión. En consecuencia, el gobierno se verá obligado a proporcionar liquidez a las instituciones financieras en dificultad. Sin embargo, al ayudar a los bancos insolventes, creará presiones inflacionarias. Si las operaciones de mercado abierto se llevan a cabo mediante una expansión de deuda interna nominal, entonces se pone en peligro la credibilidad del banco central y, por tanto, la sostenibilidad antiinflacionaria (Calvo, 1991).

Generalmente, las crisis bancarias y monetarias tienen un origen común en la desregulación del sistema financiero, los ciclos de expansión de los precios de la vivienda y las burbujas de activos (Kaminsky y Reinhart, 1999). De estos tres aspectos, la desregulación del sistema financiero ha estado siempre en el foco del debate, y muchos académicos apoyan o rechazan esta hipótesis. Kaminsky y Reinhart (1999), por ejemplo, argumentan que la liberalización financiera precedió a la mayoría de las crisis históricas. Dicha relación causal se da porque con la liberalización los movimientos de capital se enfocan en buscar ganancias a corto plazo a cambio de mayor riesgo. Este mayor riesgo se manifiesta en una evaluación menos rigurosa a los deudores por parte de los acreedores. Al final, los agentes y los bancos se ven afectados igualmente por los episodios de impago cuando explota la burbuja de crédito, movida por las expectativas de los agentes en cuanto a los precios más altos de los activos. Otra consecuencia de los mercados desregulados la sufren, en su mayoría, los países emergentes. La mayor parte del endeudamiento posterior a la liberalización tiende a ser sesgada hacia vencimientos más cortos y a estar denominada en moneda extranjera (Reinhart, 2015). Estos tipos de contratos dejan desprotegidas a estas naciones ante sus acreedores internacionales, cuando sufren cualquier revés económico.

Otro aspecto ignorado de la liberalización y la desregulación es el problema de agencia ${ }^{6}$ entre

$6 \quad$ Esta situación se da debido a la presencia de información asimétrica, donde una agente puede tomar ventaja de otro. 
el propietario (principal) y el administrador (agente) de las corporaciones y empresas modernas. En el periodo 1920-1980, las principales empresas estadounidenses tenían una propiedad dispersa entre miles de accionistas sin participación decisiva. Desde ese momento, los intereses de los propietarios (accionistas) no estaban alineados con los intereses de los administradores, y los dividendos se convirtieron en un costo más para las empresas. Con la desregulación y liberalización de los ochenta, los fondos de inversión, los fondos mutuos, las compañías de seguros y los fondos de pensiones se convirtieron en los accionistas mayoritarios en la mayoría de grandes empresas en el escenario mundial. La solución que se dio al problema de agencia en este contexto fue premiar a los administradores con ingresos extraordinarios que alinearan los intereses de los propietarios y la empresa. Lorente (2018) argumenta en este sentido:

Gran parte de estos ingresos extraordinarios de los administradores se deben a la asignación de opciones de compra de acciones, muchas veces sin relación alguna con los resultados de la empresa, con la simple justificación de que volver accionistas a los ejecutivos hará que sus intereses personales coincidan con los del resto de los accionistas. En la práctica, esta modalidad de remuneración lleva a privilegiar estrategias que aumentan el valor en Bolsa de las acciones en el corto plazo, sin preocuparse por las consecuencias de largo plazo para la supervivencia o el crecimiento de la empresa. Por ejemplo, utilizar recursos de la empresa para recomprar sus propias acciones sugiere que las utilidades se repartirán entre menos accionistas, así que la expectativa de dividendos valoriza esas acciones, pero esta estrategia reduce los recursos asignables a innovación y crecimiento. (p. 274)

Otros autores defienden la liberalización financiera y los mercados desregulados, y argumentan que las crisis financieras no son el resultado de un proceso de liberalización financiera. Las crisis reflejan la tendencia de los gobiernos para ejecutar políticas fiscales y monetarias incompatibles con la flotación del tipo de cambio, la estabilidad financiera y la disciplina del mercado (Bordo et al., 2001). Esta postura argumenta que las acciones del gobierno causan fallas de mercado y modifican las decisiones de los agentes. Cuando los agentes del mercado financiero tienen la creencia de que los funcionarios van a intervenir para protegerlos, están dispuestos a asumir riesgos excesivos (Reinhart, 2015). Ya sea la liberalización o la intervención del gobierno, la toma de decisiones pensando en ganancias a corto plazo es la norma a la hora de gestar una crisis financiera. Stiglitz (2009) señala a este respecto:

La realidad es que tanto en los Estados Unidos como en Europa occidental se ha hecho saber a las grandes empresas que, si tienen problemas, los poderes públicos las salvarán; o que, al menos, procurarán acudir a socorrerlas. Ello ha fortalecido la inclinación de esas empresas a asumir grandes riesgos, porque si los asumen y pierden serán los contribuyentes quienes pagarán las pérdidas; pero, si ganan, los beneficios serán para ellas. (p. 3)

Debido a este comportamiento del gobierno, grandes bancos vieron la oportunidad de obtener mayores ganancias a cambio de un mayor riesgo durante la crisis financiera del 2008. Sus ganancias eran privadas, pero los costos por rescatarlos fueron para toda la sociedad. La única forma de evitar estos incentivos perversos es fortalecer los diferentes marcos regulatorios y procedimientos en el sistema financiero. Los sistemas bancarios se pueden fortalecer mejorando sus respuestas ante marcos regulatorios que premian la toma de riesgo imprudente (Caprio y Klingebiel, 1997).

Vale la pena discutir el aspecto político de la desregulación financiera en los países emergentes, que confían en que el Fondo Monetario Internacional (FMI) tiene claridad sobre cuáles son las mejores prácticas en términos monetarios, financieros y fiscales. Sin embargo, los consejos 
dados por el FMI generalmente están sesgados hacia políticas en las que se minimice la injerencia del Estado en cualquier mercado, sin analizar las características institucionales de cada país. Lastimosamente, los consejos del FMI no son solo consejos, sino lineamientos que debe seguir un país para continuar consiguiendo financiamiento externo. Es así como el poder de negociación de los países emergentes es nulo ante el sistema financiero mundial, no hay posibilidad de debate teórico o metodológico, y las políticas establecidas por organismos internacionales se aplican como si todos los países fueran iguales. La mayoría de países emergentes ha puesto en marcha políticas de liberalización orientadas al mercado, para lograr un "capitalismo al estilo estadounidense" (Krugman, 2008; Stiglitz, 2011; Raworth, 2017).

Aunque sea complicado, se debe ver cada economía desde una perspectiva histórica que permita analizar la evolución de la interacción entre sus instituciones y mercados, en clave de lograr entender su nivel de desarrollo actual. Chang (2013), economista especializado en desarrollo económico, llegó a la conclusión de que los países hoy desarrollados nunca practicaron el libre comercio cuando eran países en desarrollo. Tal vez sea necesario que los países en vía de desarrollo generen sus propias políticas, para tener un sistema financiero estable y una economía real que mantenga buenas tasas de crecimiento. Chang (2013) sostiene en esta línea:

Aunque las protecciones arancelarias eran en muchos países un componente dominante de esta estrategia, no siempre eran la única medida proteccionista ya que a menudo iban acompañadas de otras medidas como subsidios a la exportación, reducciones arancelarias para los insumos usados en los productos para la exportación, asignación de derechos de monopolio, asociaciones de fabricantes, créditos dirigidos, planeamiento de la inversión y de la fuerza de trabajo, ayudas de $1+D$ y creación de instituciones para facilitar la cooperación entre los sectores público y privado. Suele pensarse que estas políticas fueron inventadas por el Japón y otros países del Este de Asia después de la Segunda Guerra Mundial, o al menos por Alemania a finales del siglo $X I X$, pero muchas de ellas tienen un largo pedigrí. (p. 21)

\section{Burbuja de crédito}

Una burbuja de crédito sucede cuando hay expectativas $^{7}$ de cambios estructurales en los mercados financieros. Estos cambios provocan un exceso de crédito que incrementa la demanda de algunos activos y, por ende, su precio sube (Glaeser, Wei Huang y Shleifer, 2017). A su vez, con un precio más alto de los activos, se incentiva aún más el crédito hasta el punto de que las entidades financieras no se preocupan por hacer una buena evaluación de los deudores. Una vez no encuentran más compradores interesados en adquirir sus activos o acciones, la burbuja explota, los precios bajan abruptamente, dejando los créditos sin pagar y llenando de riesgo y volatilidad el sistema financiero. Paradójicamente, una burbuja especulativa no desplaza las inversiones en el sector real, sucede lo opuesto: cuando los especuladores obtienen ganancias por vender sus activos valorizados, sus demandas por bienes de consumo y bienes duraderos aumentan, lo cual hace que se genere un auge de inversiones en el sector real ${ }^{8}$. El problema vuelve a darse cuando la burbuja explota, como bien expone Lorente (2018):

En ese momento, ante la ausencia de nuevos préstamos que sostengan el ritmo de pagos a los bancos, tanto los hogares como las empresas que participaron de estos excesos tienen que enfrentar sus obligaciones financieras con sus ingresos normales, es decir, con los que permitían

\footnotetext{
$7 \quad$ Las burbujas especulativas y las crisis financieras no tienen cabida en el análisis de equilibrio, ni son compatibles con la racionalidad, así que no existen para los modelos EDGE.

8 La inversión y el consumo deben crecer conjuntamente en una economía saludable.
} 
sostener sus gastos familiares o de producción. Esto frena el consumo de bienes duraderos y luego disminuye el resto de los consumos; los inventarios invendibles aumentan y las empresas reducen su producción. Crece entonces el desempleo y comienza la crisis económica que, casi de inmediato, se complica con una crisis bancaria y un racionamiento del crédito que prolongan la recesión por muchos meses. (p. 366)

Las autoridades monetarias enfrentan la disyuntiva de no intervenir en la burbuja o de hacerla "explotar" deliberadamente; la respuesta común en la comunidad académica dice que es más eficiente no intervenir. Mishkin (2017) enumera cinco razones principales por la cuales no se debería hacer "explotar" o intervenir una burbuja de activos: a) las burbujas son difíciles de detectar; b) subir la tasas de interés no afectará el comportamiento de los participantes de ese mercado, ya que estos esperan retornos muy altos por los activos de la burbuja; c) hay muchos tipos de activos y no todos están relacionados con la burbuja; d) existen opiniones de expertos que argumentan que subir los tipos de interés hace que las burbujas exploten gravemente; e) la política monetaria tiene una excelente capacidad de mantener los efectos dañinos de la burbuja a niveles manejables después de que explote.

Por su parte, Bernanke y Gertler (2001) explican que los cambios en los precios de los activos deberían afectar a la política monetaria solo si estos inciden en el pronóstico de la inflación del banco central. Entonces, la política monetaria solo deberá intervenir en las burbujas bajo unas condiciones muy específicas; una de ellas tiene lugar cuando la perturbación en la bolsa de valores cambia la tasa de interés natural de la economía.

Un posible camino que podría tomar la autoridad monetaria es bajar la tasa de interés con el objetivo de disminuir el rendimiento de los activos, para de esta forma incentivar a las personas a aumentar su demanda de dinero. Sin embargo, bajas tasas de interés pueden incrementar los incentivos a los agentes del mercado para buscar más rentabilidad a cambio de más riesgo, al tiempo que distorsionan los procedimientos de valuación de créditos bancarios (Mishkin, 2017).

Estas situaciones son las que ponen en aprietos a la autoridad monetaria, que sabe que la burbuja va a estallar eventualmente y no tiene ningún mecanismo parar evitar tal situación. Aun así, bajo estas condiciones tan desfavorables, la autoridad monetaria tiene otro camino de acción, distinto al de esperar: si sabe con certeza que el auge del mercado de valores no es impulsado por los fundamentales de la economía, y sabe exactamente cuando la burbuja va a estallar, el banco central podrá hacer una verdadera política óptima (Bernanke y Gertler, 2001). Bajo las condiciones anteriores, el banco puede evitar los efectos dañinos de la burbuja antes de que explote; sin embargo, el mercado financiero se caracteriza por sus asimetrías en la información y por la importancia de las expectativas de los agentes; así, debido a estas características, muy difícilmente el banco sabrá cuándo y cómo actuar.

El anterior panorama suena más desalentador de lo que en verdad es. Si bien es muy improbable que un banco central pueda evitar los efectos dañinos de las burbujas de crédito, se pueden hacer muchos más procedimientos para evitarlas. La combinación de políticas macro- y microprudenciales son el camino para evitar altos niveles de inestabilidad en el mercado financiero. Las políticas microprudenciales agrupan la supervisión a las instituciones financieras, requerimientos de liquidez $y$, en general, cualquier regulación que garantice el buen comportamiento financiero de cada agente individual a nivel microeconómico (Mishkin, 2017).

Muchos sistemas financieros nacionales cuentan con buenas prácticas a nivel microeconómico, donde los agentes no toman riesgos irracionales e innecesarios. A pesar de esto, la clave está en garantizar también un buen comportamiento de los agentes a nivel macroeconómico. Las interacciones entre firmas financieras pueden generar externalidades que pongan en riesgo la estabilidad del sistema financiero (Mishkin, 2017). Esta es la 
función de las políticas macroprudenciales: mitigan el riesgo sistémico de todo el sistema como un todo. Un ejemplo de política macroprudencial moderna son los requisitos de capital anticíclicos, que sirven para evitar situaciones de insolvencia en periodos de desaceleración. Con lo anterior se concluye que la regulación es necesaria en el sistema financiero, como Stiglitz (1998) argumenta:

Los mercados de capital, en particular, requieren estándares de auditoría acompañados de sistemas legales que desincentiven el fraude, provean a los inversores con información adecuada sobre los activos y pasivos de las empresas, y protejan a los accionistas minoritarios. Pero la transparencia por sí misma no es suficiente, en parte porque inevitablemente la información es imperfecta. Un buen marco legal combinado con regulación y supervisión resulta necesario para mitigar estos problemas informacionales y fomentar las condiciones para que se den mercados financieros eficientes. La regulación en los mercados financieros exitosos está al servicio de cuatro propósitos: mantener la seguridad y la robustez (regulación prudencial), promover la competencia, proteger a los consumidores, $y$ asegurar que los grupos desfavorecidos tienen algún acceso al capital. (p. 29)

\section{Contagios o derrames}

Un contagio es un movimiento conjunto excesivo de las variables económicas fundamentales en un grupo de países como consecuencia de una perturbación común (Kaminsky, Reinhart y Végh, 2003). Los contagios financieros son los que realmente producen pánico en el ámbito mundial y tienen la capacidad de hundir a regiones enteras en profundas recesiones. A pesar de su temible capacidad para poner en peligro la economía mundial, no es común que toda crisis financiera doméstica termine en un contagio financiero regional.

Kaminsky, Reinhart y Végh (2003) denominan como la impía trinidad del contagio financiero a una serie de elementos claves que producen contagios financieros: una reversión abrupta de los flujos de capital, anuncios sorpresa y un acreedor común con un alto nivel de apalancamiento (cociente entre activos y capital). Solo después de que se den estas condiciones, se puede reconocer la transmisión de una crisis financiera desde un país hacia otros a través de los mercados financieros globales. Un ejemplo de este mecanismo fue la crisis de la deuda latinoamericana, cuando en 1982 México anunció que no podía seguir pagando sus obligaciones a sus acreedores internacionales. Esto limitó la confianza de los acreedores comunes hacia los demás países de la región y restringió el crédito para la mayoría. Cuando un país mantiene un déficit considerable en cuenta corriente y depende fuertemente de préstamos a corto plazo para su financiamiento, los ajustes necesarios serán muy difíciles de afrontar cuando las entradas de capital se agoten (Bordo et al., 2001).

Los canales de contagio de una crisis también pueden tomar la forma de perturbaciones en las variables reales. Se debe tener en cuenta que cuando hay apertura en los mercados de bienes, los consumidores y empresas gastan parte de su ingreso disponible en bienes extranjeros (Blanchard et al., 2012). Durante una crisis financiera, la renta disponible de los habitantes de un país disminuye $y$, por ende, las importaciones que realiza; esto provoca una disminución en el ingreso y en la producción real de los países con los que tiene relaciones comerciales. Este canal de contagio toma la forma de un proceso de retroalimentación positiva en el cual el deterioro de la producción y del comercio se expande a medida que un nuevo país se ve afectado por la cadena de dificultades en las relaciones comerciales regionales; muy rápidamente una crisis originada en un lugar específico se ha expandido a nivel regional. 


\section{CONSECUENCIAS Y DATOS EMPÍRICOS}

Reinhart y Rogoff (2008) muestran que para las cinco crisis más profundas en economías avanzadas después de la Segunda Guerra Mundial (Finlandia, Japón, Noruega, España y Suecia), la caída en el crecimiento anual de la producción superó el $5 \%$ y el crecimiento se mantuvo por debajo de la tendencia precrisis incluso después de tres años. La evidencia empírica sobre el impacto que tienen las crisis financieras demuestra la preocupación de la comunidad académica por crear un marco teórico y normativo más fuerte. La sociedad civil es la más afectada por esta clase de acontecimientos; sus consecuencias son especialmente notables en el nivel de empleo de la economía después de la crisis, pues la tasa de desempleo se eleva un promedio de 7 puntos porcentuales con respecto al promedio del ciclo económico (Reinhart y Rogoff, 2009).

Con el sistema económico de un país sumido en una profunda recesión, los altos niveles de desempleo sostenidos son solo una consecuencia lógica. Una economía avanzada que vivió una profunda crisis financiera fue Suecia, que tuvo una crisis monetaria y bancaria en 1990. Entre 1990 y 1994, este país tuvo pérdidas de empleo de 16,6 puntos porcentuales (Jonung y Hagberg, 2005). Al igual que Suecia, Finlandia sufrió una crisis bancaria y monetaria en 1990 como consecuencia de la explosión de una burbuja de crédito motivada por unos precios altos de los activos. Entre 1990 y 1994, la pérdida acumulada de empleo fue de 24,0 puntos porcentuales. El ingreso real finlandés no volvió a su anterior tendencia hasta 1994 y perdió 26,4 puntos porcentuales acumulados (Jonung y Hagberg, 2005).

De la evidencia anterior es notable resaltar que las consecuencias en el nivel de desempleo y de ingreso son más duraderas y profundas en economías avanzadas que en economías emergentes. Esta diferencia se debe a que los países emergentes tienen una mayor flexibilidad de los salarios hacia abajo que los países avanzados (Reinhart y Rogoff, 2009). La razón de esta particular situación se debe a que los países avanzados tienen usualmente mejores sistemas de seguridad social, mayores niveles de ahorro, sindicatos más fuertes y un mejor nivel de vida que los países en vía de desarrollo (Krugman, 2008). Por las anteriores razones, los trabajadores de economías avanzadas pueden esperar más tiempo por un empleo con salarios más aceptables, a diferencia de los trabajadores de economías emergentes, que se ven obligados a aceptar salarios muy bajos.

Los costos fiscales de una crisis deben ser vistos como transferencias de los contribuyentes a un grupo específico de actores (industrias, bancos comerciales y otros tipos de instituciones financieras) dentro de la economía (Jonung y Hagberg, 2005). En secciones anteriores se demostró que el gobierno se ve obligado a implementar estabilizadores fiscales automáticos en tiempos de recesión para intentar sacar la economía a flote. Esta medida es necesaria, pero los estímulos fiscales aumentan el déficit del gobierno en un entorno en el que el ingreso tributario disminuye. La combinación de bajas en el ingreso tributario, deterioro en las variables fundamentales de la economía y aumentos en el gasto público son las principales razones por las cuales muchos gobiernos y países se han visto a entrar en default después de una crisis financiera. La implementación de estabilizadores fiscales automáticos, políticas fiscales anticíclicas y el pago de intereses más altos debido a reducciones en las calificaciones crediticias conducen a un déficit fiscal mayor (Reinhart y Rogoff, 2011).

Un panorama aún más desalentador es el hecho de que los estímulos fiscales no son suficientes para estimular rápidamente la economía y sacarla a flote. Después de una crisis financiera, el precio real de la vivienda disminuye en promedio $35 \%$ por seis años y el capital para inversión cae en promedio $55 \%$ por tres años y medio (Reinhart y Rogoff, 2009).

\section{CONCLUSIONES}

Este artículo solo presenta una pequeña porción de toda la discusión que rodea a una crisis financiera; 
sin embargo, se demostró que los modelos macroeconómicos actuales se quedan cortos para explicar las interacciones dentro los mercados financieros; por consiguiente, se necesita la construcción de un marco metodológico y teórico más amplio. Enfoques como la economía de la complejidad y la unión de la economía con la psicología pueden ayudar a dar un paso hacia delante. En segundo lugar, se demostró que un banco central está muy limitado para actuar contra burbujas de crédito, y la clave para evitarlas está en crear marcos regulatorios más eficaces, no en hacerlas explotar.

Blanchard, Dell'Ariccia y Mauro (2010) muestran que, a la hora de combatir una recesión, las medidas fiscales discrecionales llegan tarde y no son inmediatas. Debido a lo anterior, se deben mejorar los estabilizadores fiscales automáticos para limitar las consecuencias en la economía; más aún, la inversión en infraestructura genera eslabonamientos productivos hacia adelante y hacia atrás capaces de crear e impulsar nuevos sectores. La infraestructura genera una red de externalidades positivas y de economías de escala que propician un mejoramiento en las instituciones y el crecimiento de los países (Esfahani y Ramírez, 2003; Bértola y Ocampo, 2012).

Anticipar una crisis financiera requiere de una extraña combinación de suerte y atención a los datos; sin embargo, este es el juego al que justamente se dedican muchos economistas. Por ejemplo, la lira turca se ha devaluado más del $40 \%$, y a este fenómeno se le suma el comportamiento del régimen autoritario de Ankara, que se niega a subir los impuestos y la tasa de interés para frenar la espiral inflacionaria. Como se ha visto, una crisis financiera nacional puede crecer muy rápidamente hasta volverse internacional; por eso, en específico la crisis turca puede poner en aprietos a las economías emergentes alrededor del mundo. Ávila (2018) indica en este sentido:

Es probable que la disponibilidad de fondos para las economías emergentes se reduzca y que las tasas de interés suban. Colombia, con acreencias externas importantes, no saldrá indemne. Y así nos hayamos portado bien, la historia nos enseña que aquí también pagan los justos por los pecadores.

Lastimosamente, la crisis turca no es lo único que amenaza a los países emergentes y a la estabilidad financiera global; la crisis argentina también podría tener consecuencias serias. El gobierno de Macri escogió el peor momento posible para "abrirse al mundo"; el proteccionismo está en auge y sus medidas han golpeado a muchas economías del mundo, especialmente a Argentina y Turquía. Al momento de escribir este artículo, Argentina tiene la tasa de interés más alta del mundo, con $60 \%$, y ha restringido el gasto público para evitar entrar en default y provocar un episodio de hiperinflación (el FMI estima una inflación del $40 \%$ para el cierre del año). El gobierno de Macri ha hecho justamente lo opuesto a lo expuesto en este artículo: la ausencia de estabilizadores automáticos y política monetaria laxa hará que salir de esta crisis sea una tarea titánica. Más aún, las condiciones con que el gobierno Macri ha obtenido crédito no son nada favorables (pagos desmesurados, tasas de interés altas y primas de riesgo elevadas) y plantean problemas serios para el país hacia futuro. Stiglitz, premio Nobel de Economía y antiguo economista en jefe del Banco Mundial, atendió una entrevista a la BBC donde respondió las siguientes preguntas: ¿hay posibilidades de un efecto contagio para el resto de la región latinoamericana?, ¿es este un problema particular de Argentina? Su respuesta no fue alentadora:

Este es un problema particular para Argentina, pero una gran crisis para Argentina podría claramente afectar a los otros países a través de varios canales. $Y$ particularmente en este momento concreto porque los países de todo el mundo con grandes déficits de cuenta corriente, como Turquía, o grandes déficits presupuestarios enfrentan problemas. Hay varios países, no voy a mencionar sus nombres, que creo que pueden estar 
maduros para una crisis. Si hay un par de ellas en un periodo corto, es un efecto multiplicador y podría crear una crisis en los mercados emergentes. Por otro lado, los problemas podrían permanecer localizados. En términos más generales, creo que hay preocupaciones". (Lissardy, 2018)
La combinación de las crisis en Turquía y Argentina, el proteccionismo de Donald Trump, la tan esperada normalidad monetaria de la Reserva Federal, el éxodo de población venezolana a distintos países latinoamericanos y el Brexit nos hacen pensar que esta vez tampoco será distinto.

\section{REFERENCIAS}

1. Ahn, T. K., Ostrom, E. y Walker, J. M. (2003). Heterogeneous preferences and collective action. Public Choice, 117(3/4), 295-314. https://doi.org/10.1023/B:PUCH.0000003739.54365.fd

2. Ávila, R. (2018, 12 de agosto). El efecto Turquía. Portafolio. Recuperado de http://www.portafolio.co/ opinion/ricardo-avila/el-efecto-turquia-brujula-ricardo-avila-519982

3. Banco Mundial (2018). Global Financial Development report 2017/2018: Bankers without Borders. Washington: Autor.

4. Bernanke, B. y Gertler, M. (2001). Should Central Banks respond to movements in asset prices? The American Economic Review, 91(2), 253-257. https://doi.org/10.1257/aer.91.2.253

5. Blanchard, O. (1997). Is there a core of usable macroeconomics? The American Economic Review, 87(2), 244-246. https://doi.org/10.1257/jep.32.1.97

6. Blanchard, O. (2018). Should we reject the natural rate hypothesis? The Journal of Economic Perspectives, $32(1), 97-120$.

7. Blanchard, O., Amighini, A. y Giavazzi, F. (2012). Macroeconomía. Madrid: Pearson.

8. Blanchard, O., Dell'Ariccia, G. y Mauro, P. (2010). Repensar la política macroeconómica. Revista de Economía Institucional, 12(22), 61-82.

9. Bordo, M., Eichengreen, B., Klingebiel, D. y Martínez-Peria, M. S. (2001). Is the crisis problem growing more severe? Economic Policy, 16(32), 51-82. https://doi.org/10.1111/1468-0327.00070

10. Calvo, G. (1991). The perils of sterilization. Staff Papers (International Monetary Fund), 38(4), 921-926. https://doi.org/10.2307/3867130

11. Caprio, G. y Klingebiel, D. (1997). Bank insolvency: Bad luck, bad policy, or bad banking? ]En M. Bruno (Ed.), Annual World Bank Conference on Development Economics 1996 (pp. 79-104). Washington: Banco Mundial.

12. Chang, H.-J. (2013). Patada a la escalera: la verdadera historia del libre comercio. Ensayos de Economía, $23(42), 27-57$.

13. Chorafas, D. (2013). Queen Elizabeth II and the economists: The changing role of Central Banks. En The changing role of Central Banks. Nueva York: Palgrave Macmillan. https://doi.org/10.1057/9781137332288

14. Colander, D., Howitt, P., Kirman, A., Leijonhufvud, A. y Mehrling, P. (2008). Beyond DSGE models: Toward an empirically based macroeconomics. The American Economic Review, 98(2), 236-240. https:// doi.org/10.1257/aer.98.2.236

15. Dornbusch, R., Fischer, S. y Startz, R. (2007). Macroeconomía (10. a ed.). Ciudad de México: McGraw-Hill.

16. Esfahani, H. S. y Ramírez, M. T. (2003). Institutions, infrastructure, and economic growth. Journal of Development Economics, 70(2), 443-477. https://doi.org/10.1016/S0304-3878(02)00105-0 
17. Farhi, E. y Tirole, J. (2018). Shadow banking and the four pillars of traditional financial intermediation [EconPol Working Paper 16]. https://doi.org/10.3386/w23930

18. Glaeser, E., Wei Huang, Y. M. y Shleifer, A. (2017). A real estate boom with Chinese characteristics. The Journal of Economic Perspectives, 31 (1), 93-116. https://doi.org/10.1257/jep.31.1.93

19. Haldane, A. y Turrell, A. (2017). An interdisciplinary model for macroeconomics [Staff Working Paper 696]. https://doi.org/10.2139/ssrn.3079789

20. Hernández, I. (2005). Teoría política y fiscal. Bogotá: Universidad Externado de Colombia.

21. Janssen, M. y Ostrom, E. (2006). Empirically based, agent-based models. Ecology and Society, 11 (2). https:// doi.org/10.5751/ES-01861-110237

22. Jonung, L. y Hagberg, T. (2005). How costly was the crisis of the 1990s? A comparative analysis of the deepest crises in Finland and Sweden over the last 130 years. Bélgica: Comisión Europea.

23. Kaminsky, G. y Reinhart, C. (1999). The twin crises: The causes of banking and balance-of-payments problems. The American Economic Review, 89(3), 473-500. https://doi.org/10.1257/aer.89.3.473

24. Kaminsky, G., Reinhart, C. y Végh, C. (2003). The unholy trinity of financial contagion. The Journal of Economic Perspectives, 17(4), 51-74. https://doi.org/10.1257/089533003772034899

25. Kirman, A. (1989). The intrinsic limits of modern economic theory: The emperor has no clothes. The Economic Journal, 99(395), 126-139.

26. Krugman, P. (2008). Trade and wages, reconsidered. Brookings Papers on Economic Activity, 2008, 103-137.

27. Lissardy, G. (2018, 6 de septiembre 6). Joseph Stiglitz, Nobel de Economía, sobre la crisis de Argentina: "Las medidas de austeridad ralentizarán la economía e impondrán un alto costo en la gente". BBC News Mundo. Recuperado de https://www.bbc.com/mundo/noticias-america-latina-45427972

28. Lorente, L. (2018). Dinámica del crecimiento económico. Bogotá: Universidad Nacional de Colombia.

29. Lucas, R. (1976). Econometric policy evaluation: A critique. Carnegie-Rochester Conference Series on Public Policy, 1(1), 19-46. https://doi.org/10.1016/S0167-2231(76)80003-6

30. Mishkin, F. S. (2017). Rethinking monetary policy after the crisis. Journal of International Money and Finance(73), 252-274.

31. Monsalve, S. (2017). Competencia bajo equilibrio general. Bogotá: Universidad Nacional de Colombia.

32. Ostrom, E. (2000). The danger of self-evident truths. PS: Political Science and Politics, 33(1), 33-44. https:// doi.org/10.2307/420774

33. Prescott, E. (1995). Comment on computable general equilibrium models and monetary policy advice. Journal of Money, Credit and Banking, 27(4), 1502-1505. https://doi.org/10.2307/2078067

34. Prescott, E. (2002). Prosperity and depression. The American Economic Review, 92 (2), 1-15.

35. Prescott, E. (2006). The transformation of macroeconomic policy and research. The American Economist, 50(1), 3-20. https://doi.org/10.1177/056943450605000101

36. Raworth, K. (2017). Doughnut economics : seven ways to think like a $21^{\text {st }}$ century. Vermont: Chelsea Green Publishing.

37. Reinhart, C. (2015). The antecedents and aftermath of financial crises as told by Carlos F. Díaz-Alejandro. Economía, 16(1), 189-217. https://doi.org/10.3386/w21350 
38. Reinhart, C. y Rogoff, K. (2008). Is The 2007 U.S. subprime crisis so different? An international historical. American Economic Review, 98(2), 339-344. https://doi.org/10.1257/aer.98.2.339

39. Reinhart, C. y Rogoff, K. (2009). The aftermath of financial crises. The American Economic Review, 99(2), 466-472. https://doi.org/10.1257/aer.99.2.466

40. Reinhart, C. y Rogoff, K. (2011). Esta vez es distinto: ocho siglos de necedad financiera. Madrid: Fondo de Cultura Económica.

41. Reinhart, C. y Rogoff, K. (2014). Recovery from financial crises: Evidence from 100 Episodes. The American Economic Review, 104(5), 50-55. https://doi.org/10.1257/aer.104.5.50

42. Sims, C. (1996). Macroeconomics and Methodology. The Journal of Economic Perspectives, 10(1), 105-120. https://doi.org/10.1257/jep.10.1.105

43. Snowdon, B. y Vane, H. (2005). Modern macroeconomics: Its origins, development and current state. Cornwall: Edward Elgar Publishing.

44. Solow, R. (2004). Even a worldly philosopher needs a good mechanic. Social Research, 71 (2), 203-210.

45. Solow, R. (2008). The state of macroeconomics. The Journal of Economic Perspectives, 22(1), 243-246. https://doi.org/10.1257/jep.22.2.243

46. Stiglitz, J. (1998). Más instrumentos y metas más amplias para el desarrollo. Hacia el Consenso Post-Washington. Recuperado de http://old.clad.org/portal/publicaciones-del-clad/revista-clad-reforma-democracia/articulos/012. https://doi.org/10.2307/3467160

47. Stiglitz, J. (2009). Crisis mundial, protección social y empleo. Revista Internacional del Trabajo, 128(1-2). https://doi.org/10.2307/3467160

48. Stiglitz, J. (2011). Rethinking development economics. The World Bank Research Observer, 26(2), 230-236. https://doi.org/10.1093/wbro/lkr011

49. Stiglitz, J. y Gallegati, M. (2011). Heterogeneous interacting agent models for understanding monetary economies. Eastern Economic Journal, 37(1), 6-12. https://doi.org/10.1057/eej.2010.33

50. Svensson, L. (2000). Open-economy inflation targeting. Journal of International Economics, 50(2000), 155-183. https://doi.org/10.1016/S0022-1996(98)00078-6

51. Thaler, R. (2000). From Homo economicus to Homo sapiens. The Journal of Economic Perspectives, 14(1), 133-141. https://doi.org/10.1257/jep.14.1.133 